\title{
Superficial Temporal Artery to Middle Cerebral Artery Bypass
}

\author{
David W. Newell, M.D.'
}

The superficial temporal artery to middle artery bypass is a technique that allows the blood supply from the extracranial carotid circulation to be routed to the distal middle cerebral artery branches. The procedure allows blood flow to bypass proximal lesions of the intracranial vasculature. The performance of this bypass requires specialized microvascular training and the use of microvascular techniques. The techniques involved in performing these procedures include microdissection of the superficial temporal artery in the scalp, microdissection of the recipient middle cerebral artery branches near the sylvian fissure, and anastomosis techniques using either microvascular sutures or a microanastomotic device. The successful completion of the bypass and subsequent patency requires meticulous attention to technical details.

KEYWORDS: Middle cerebral artery, superficial temporal artery to middle cerebral artery bypass, cerebral revascularization

The superficial temporal artery to middle cerebral artery (STA-MCA bypass) can be used to augment flow to the distal MCA vascular territory. Technical developments in microvascular suture material, operating microscopes, and microsurgical techniques allowed the developments of this procedure. The technique was first described by M.G. Yasargil, who perfected the use of microvascular anastomosis in dogs. ${ }^{1}$ The original technique described was an end-to-side anastomosis using a distal branch of the STA dissected from the scalp to a surface cortical branch of the MCA near the region of the sylvian fissure (Fig. 1). Subsequent variations of this procedure have been described, including double-limb STA-MCA bypass grafts for additional flow, and end-to-end anastomoses, using a microanastomotic device for higher-flow bypasses.

\section{HISTORICAL ASPECTS}

The first STA-MCA bypass was performed in a human by M.G. Yasargil on October 30, 1967, in Zurich, on a patient with a complete occlusion of the MCA. ${ }^{1}$ Before performing this operation,

Skull Base, volume 15, number 2, 2005. Address for correspondence and reprint requests: David W. Newell, M.D., Seattle Neuroscience Institute, 1600 East Jefferson St., Ste. 620, Seattle, WA 98122. E-mail: david.newell@swedish.org. ${ }^{1}$ Seattle Neuroscience Swedish Hospital Medical Center, Seattle, Washington. Copyright (C) 2005 by Thieme Medical Publishers, Inc., 333 Seventh Avenue, New York, NY 10001, USA. Tel: +1(212) 584-4662. 1531-5010,p;2005,15,02,133,142,ftx,en;sbs00446x. 


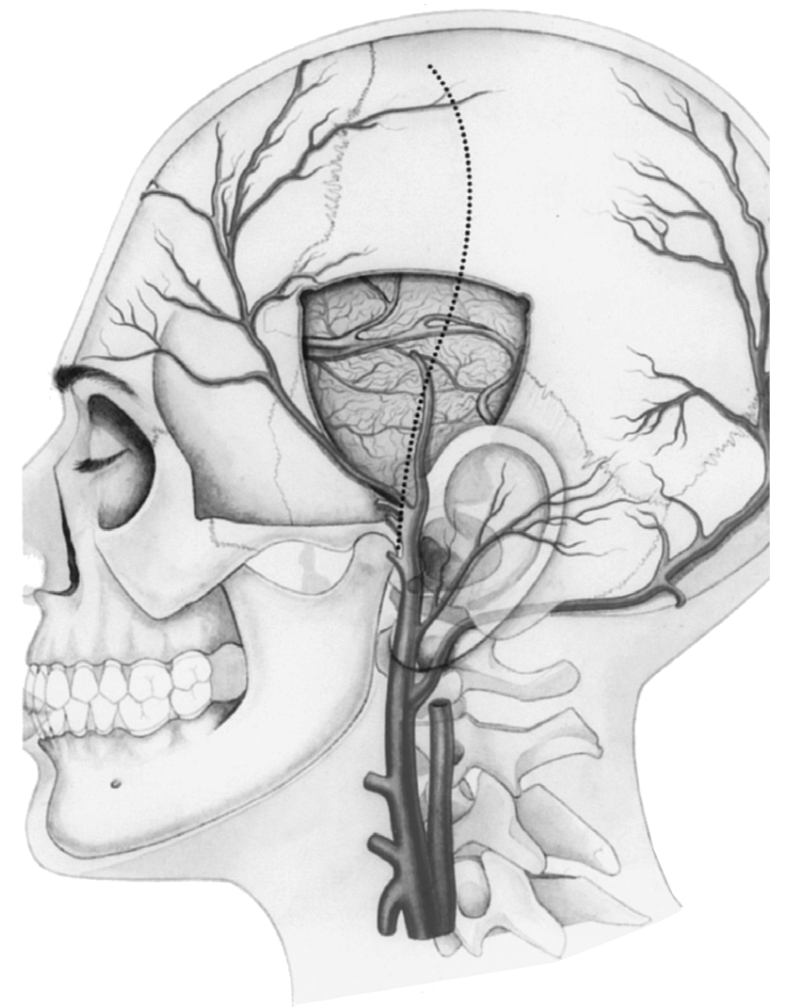

Figure 1 Schematic representation of the STA-to-MCA anastomosis using the parietal branch as a donor artery. STA, superficial temporal artery; MCA, middle cerebral artery.

Professor Yasargil studied the technique of microvascular reconstruction in small animals with R.M.P. Donaghy at the University of Vermont. The use of bipolar coagulation and the availability of microvascular sutures and the use of the operating microscope were important technical developments that enabled this procedure to be performed. The first publication of a series of patients with STA-MCA bypasses was for cerebrovascular occlusive disease and also as an adjunct in the treatment of complex intracranial aneurysms. ${ }^{1}$ Several subsequent publications appeared documenting the safety and results of STA-MCA bypass for a variety of conditions, most predominantly cerebrovascular occlusive disease. ${ }^{2-6}$ The rationale for its use in occlusive disease was based on the fact that experimental studies had identified areas of hypoperfusion following a proximal artery obstruction in cerebral circulation. At this time, before endovascular cerebrovascular techniques were introduced, there was no other acceptable treatment for narrowing in the carotid artery high above the carotid bifurcation or in the proximal middle cerebral vasculature. The widespread use of the procedure for cerebral vascular disease led to an international randomized study of STA-MCA bypass versus medical therapy for atherosclerotic lesions of the carotid artery distal to the carotid bifurcation, carotid occlusion, and lesions of the MCA. There were broad entry criteria for patients, and hemodynamic measurements were not performed in patients prior to entry. The results of the trial indicated that STA-MCA bypass was not superior to medical therapy for treatment of these lesions. ${ }^{7}$ The publication of this study led to a dramatic decrease in the number of STA-MCA bypasses performed for cerebrovascular occlusive disease.

Over the next several decades, the procedure was most commonly performed as an adjunct in the treatment of complex aneurysms requiring additional blood supply to the middle cerebral territory for occlusion, in patients with moyamoya disease, and in patients with recurrent disabling transient ischemic attacks on a hemodynamic basis. ${ }^{8-11}$ The procedure has also been used in patients with skull base tumors who require proximal vessel sacrifice as part of their treatment. More recent studies on the natural history of high stroke risk in patients with symptomatic carotid occlusion and hemodynamic insufficiency have indicated a strong rationale for re-examining the procedure for this subgroup of patients. These findings have led to the initiation of the carotid occlusion surgery study (COSS), which is currently recruiting patients. ${ }^{12}$ This study will examine the role of STA-MCA bypass in patients with symptomatic carotid occlusion who have evidence of cerebral perfusion abnormalities based on positron emission tomography scan. Patients are randomized to either bypass or medical treatment, and the efficacy of their procedure for stroke prevention is being examined.

Other indications for STA-MCA bypass have included several conditions that could 
interrupt the blood flow to the distal middle cerebral vasculature. These conditions include planned proximal vessel sacrifice for tumors or aneurysms, moyamoya disease, and other causes of vascular occlusion that reduce blood flow to the MCA territory. It was apparent from the cooperative study on aneurysms that the STA-MCA bypass is a technically feasible procedure with a high patency rate and the ability to provide distal blood flow to the cerebral vasculature. Improvements in cerebral blood flow measurement techniques, as well as the routine use of temporary balloon occlusion techniques, has led to an improvement in the capability of preoperative diagnostic testing to predict patients who may benefit from the procedure for certain indications such as ischemia and planned vessel sacrifice.

\section{PREOPERATIVE ASSESSMENT OF PATIENTS UNDERGOING STA-MCA BYPASS}

Standard preoperative imaging of patients to undergo STA-MCA bypass includes computed tomography scan as well as four-vessel cerebral angiography with selective external runs to define the STAs. The STA commonly bifurcates in the preauricular region into a frontal and parietal branch (Fig. 2). The frontal branch commonly

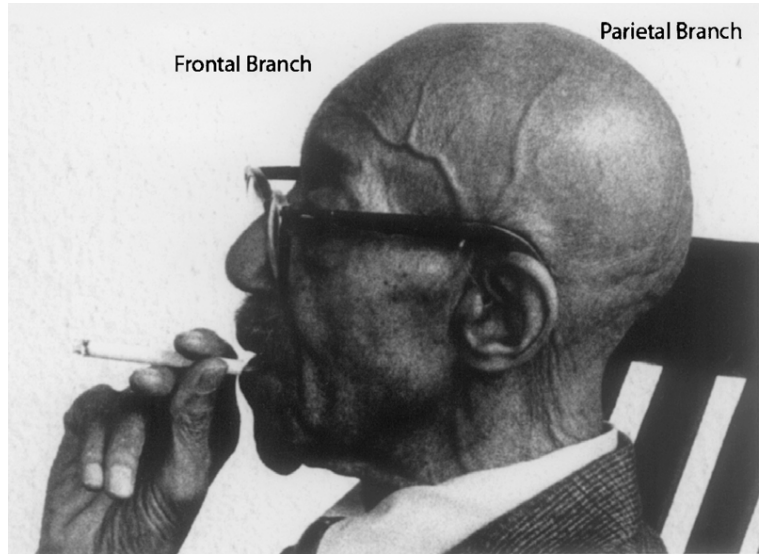

Figure 2 Photograph of an individual with a very prominent STA demonstrating the anatomy of the frontal and parietal branches (labeled). STA, superficial temporal artery. anastomoses with branches of the supraorbital artery. Variations in the anatomy include one of the two branches being dominant, equal caliber of both branches, or atresia of the parietal branch. In patients who have had a previous craniotomy, the parietal branch is often absent. The operation is quite difficult to perform if the donor artery branch of the STA is less than $1 \mathrm{~mm}$ in diameter.

Lesions of the skull base such as tumors, which produce gradual occlusion of the proximal internal carotid artery, may often lead to hypertrophy of the frontal branch of the STA as it provides collateral flow through the supraorbital pathways, which anastomose with the ophthalmic artery to provide retrograde flow into the internal carotid artery. If the frontal branch is chosen for use, it is important to note that it is usually in front of the hairline and is often crossed by branches of the superior division of the facial nerve supplying the frontalis muscle. Interruption of these branches of the facial nerve will commonly cause a partial or complete frontalis muscle weakness. Options for dissection of the frontal branch of the STA include direct dissection over the artery, or from the underside of the scalp flap with identification of the main trunk of the artery proximally, and dissection of the STA from the underside of the scalp flap distally. The second approach is preferred by the author.

\section{ANESTHETIC CONSIDERATIONS}

General anesthesia usually consisting of a combination of isoflurane and intravenous agents is used. Ventilation is adjusted to maintain the arterial $\mathrm{pCO}_{2}$ between 35 to $40 \mathrm{~mm} \mathrm{Hg}$. Mannitol is not given and a cerebrospinal fluid drain is not used to prevent retraction of the cortex from the craniotomy site and stretching of the graft. Mild hypothermia is used for cerebral protection. Most recently we have employed the Innercool (Innercool Therapies, Inc., San Diego, CA) catheter for rapid induction of hypothermia to 33 to 34 degrees Centigrade for temporary vessel occlusion and rapid rewarming 
following temporary cross-clamping. Slight elevation in arterial pressure during arterial crossclamping is also utilized to protect against cerebral ischemia.

\section{OPERATIVE POSITIONING}

The patient is placed in a supine position with a roll under the shoulder to elevate the side of the surgery, and an incision is marked directly over the STA branch identified by Doppler ultrasound (Fig. 3). If the frontal branch is also used, an L-shape flap is outlined to allow retraction of the scalp for dissection of the frontal branch from the underside of the galeal layer.

\section{EXPOSURE OF STA}

Options to expose and isolate the STA branch, or branches, include excising the scalp over the artery with a scalpel and dissection of the artery using a jeweler's forceps for cauterization of side branches, or use of needle-point cautery. The author has converted to needle-point cautery, which provides

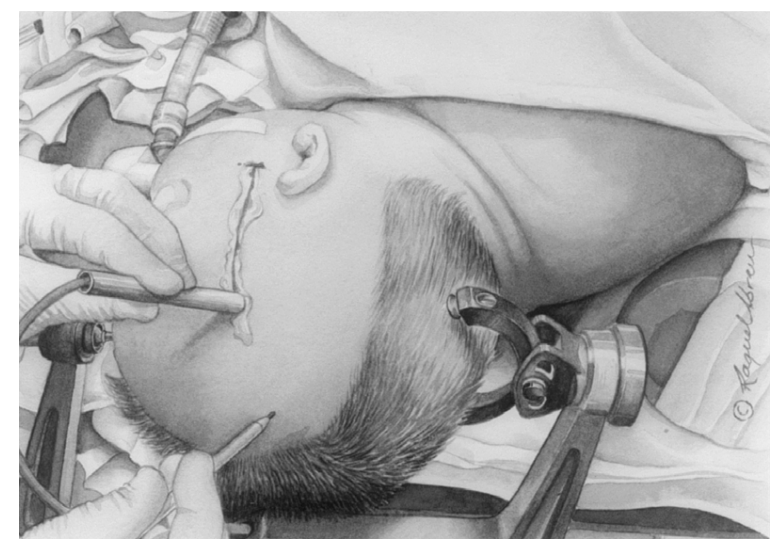

Figure 3 The patient is positioned so the head can be turned to align the temporal and parietal region parallel to the floor. The parietal branch of the STA is identified using Doppler ultrasound and marked. STA, superficial temporal artery.

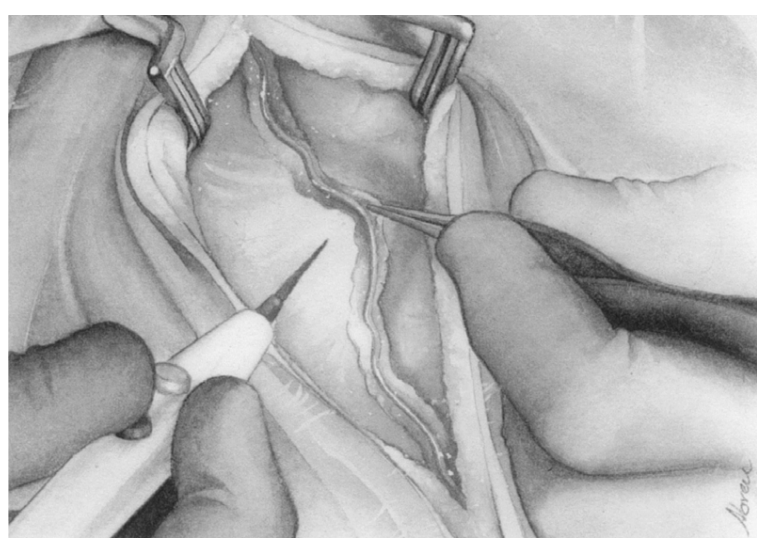

Figure 4 Illustration of the technique of dissection of the STA using needle-point cautery. Initial dissection of the scalp is performed directly over the artery and then the galeal layer is cauterized parallel to the artery to dissect it from the scalp and seal any small side branches. STA, superficial temporal artery.

an almost bloodless dissection of the artery from the surrounding tissues and easily allows effective cautery of side branches (Fig. 4). Following the dissection of an appropriate length of STA, the artery is irrigated with heparinized saline and then crossclamped with a temporary clip at the distal end. The artery is then mobilized from the galeal layer over the temporalis muscle then stored in wet gauze.

\section{DISSECTION OF FRONTAL BRANCH OF THE STA}

In cases where two limbs of the STA are needed, or under circumstances where it is determined that the parietal branch of the STA is too small for suitable use, the frontal branch of the STA can be dissected. In preparation for a frontal branch dissection, the author prefers to make an additional incision in the scalp at a right angle to the initial frontoparietal incision, which courses toward the supraorbital ridge and stops at the hairline. This allows mobilization of the scalp flap forward to expose the frontal branch of the STA on the underside of the galea layer. The dissection of the STA is taken proximally until the bifurcation is identified and then the STA is followed and dissected with tenotomy scissors and 


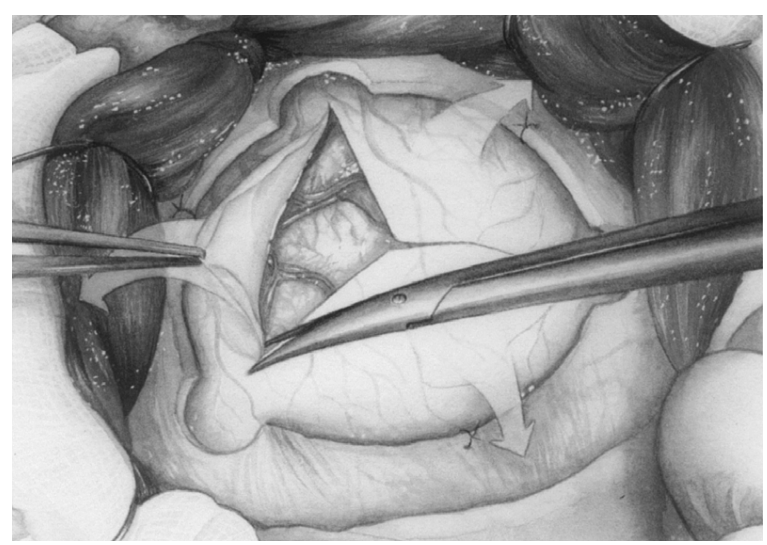

Figure 5 Technique and location of dural opening illustrating the exposure of the cortex over the distal portion of the sylvian fissure. The graft is passed through the most inferior aspect of the dural opening.

needle-point cautery. After a sufficient length of STA is dissected, it is then divided, irrigated, and cross-clamped with a temporary clamp on the distal portion of the artery.

Following dissection and isolation of the donor artery or arteries, the temporalis muscle is divided using cautery, dissected from the underlying bone, and retracted laterally on both sides of the incision. The cranial flap is then placed, which is centered $\sim 6 \mathrm{~cm}$ superior to the external auditory meatus. This location allows access to the branches of the MCA as they emanate from the distal portion of the sylvian fissure. The dura is then opened in a Y-shaped fashion and dural tack-up stitches are placed (Fig. 5). The cortex is then inspected and a suitable branch of the MCA M-4 branches are located. It is important to identify a recipient branch free from major side branches and easily dissectible. The microscope is then brought into position in the arachnoid membrane directly overlying the chosen recipient branches dissected from the artery using a combination of a jeweler's forceps and microscissors (Fig. 6). The jeweler's bipolar cautery is utilized to cauterize small cortical branches that tether the artery to the underlying cortex. The artery is mobilized for $\sim 2 \mathrm{~cm}$, and a rubber barrier is placed under the recipient artery for isolation (Fig. 7).

At this stage the donor branch is then brought into the field on a cotton patty and micro-

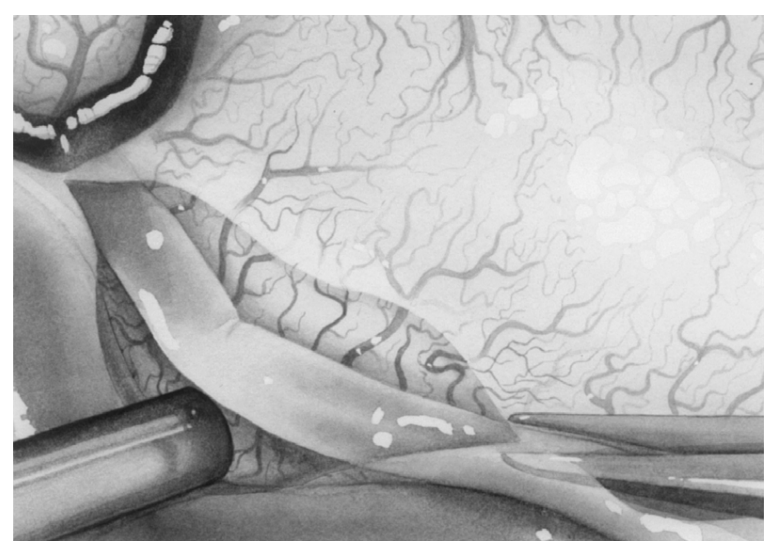

Figure 6 Illustration of the technique of exposure of the cortical recipient branch of the MCA. The arachnoid layer is carefully teased off with a combination of sharp dissection and dissection with jeweler's forceps. MCA, middle cerebral artery.

dissection of the distal end of the STA is performed. The surrounding tissue is dissected from a 1- to 2$\mathrm{cm}$ portion of the artery to free all tissue, except for the adventitia. This allows a working length of the artery for anastomosis. Following dissection of the STA, a dilute solution of papaverine $(15 \mathrm{mg}$ per 100 cc of $0.9 \%$ saline) is placed on the donor artery and recipient artery to mitigate vascular spasm. The STA is then divided at an angle and spatulated to prepare it for anastomosis.

In preparation for cross-clamping of the recipient artery, the anesthesiologist is asked to

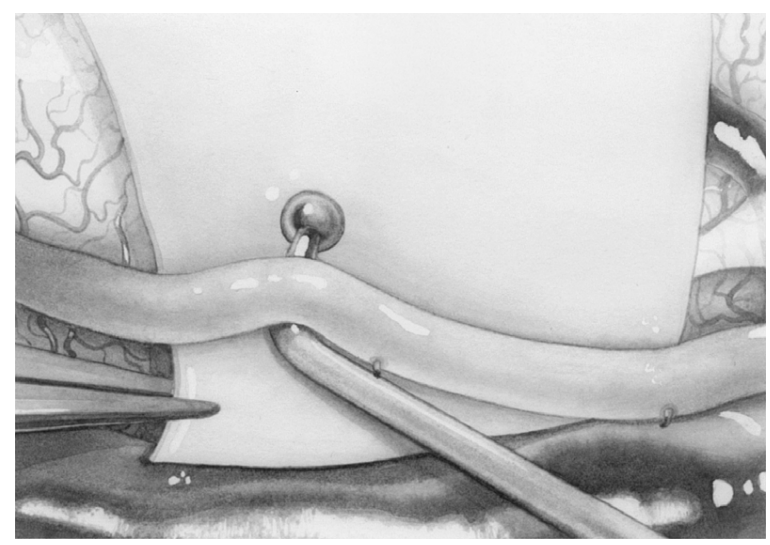

Figure 7 It is necessary to cauterize and divide multiple tiny perforating branches to the cortex to mobilize the recipient artery and place a small rubber working surface to perform the arteriotomy and anastomosis. 
elevate the blood pressure and confirm that the patient's temperature has been lowered to between 33 and 34 degrees Centigrade. Cerebral metabolic activity can also be reduced with barbiturates or propofol anesthesia. A combination of these maneuvers may be beneficial to provide cerebral protection during cross-clamping of the recipient cortical artery during the anastomosis procedure, which usually takes between 25 and 40 minutes.

Small temporary clips are then placed across the MCA recipient branch to interrupt blood flow. Either "Medhorn Beamer" or a small "Aesculup" temporary clips can be used for this purpose. Following cross-clamping, a gently curved Van Ness Scissors is used to perform the small arteriotomy. The arteriotomy length should be approximately twice the diameter of the recipient artery. It is important to try to match this length with the length of the arteriotomy and spatulated donor artery. Once the vessels are prepared, a 10-0 nylon microsuture on a noncutting needle is used to place end stitches at the heel and the end of the spatulated artery (Fig. 8). Following the placement of the heel and the sutures, either an interrupted or a running suture array can be placed at either edge of the arteriotomy (Fig. 9). After completion of the arteriotomy, the temporary clamps are removed from the recipient and donor vessels, and flow is established. A small amount of leakage of blood at the suture site is common and can be mitigated with an application

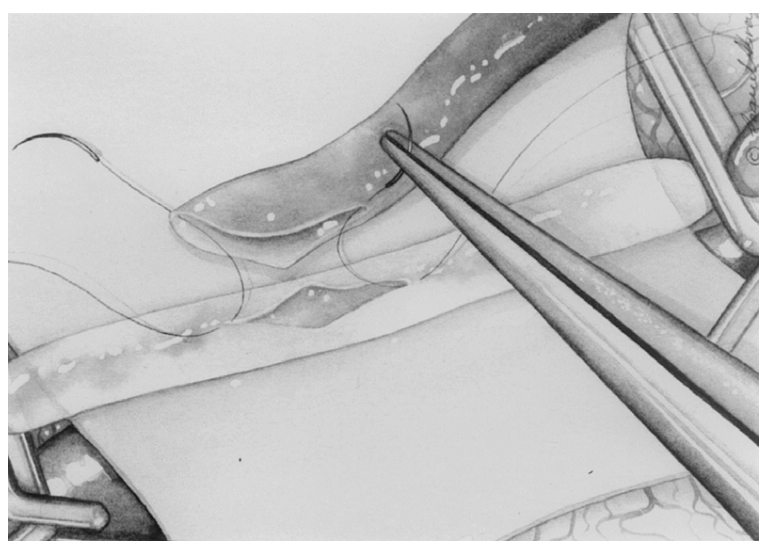

Figure 8 Placement of the end sutures aligns the donor and recipient arteries for the remainder of the anastomosis. 10-0 sutures with a noncutting needle are used.

of a small amount of Surgicel and a microcottonoid patty. If a significant jet of blood continues at the anastomotic site, additional interrupted sutures can be placed.

Microvascular Doppler ultrasound is in use to confirm patency of the anastomosis. Flow velocity signal throughout the cardiac cycle through diastole is essential in predicting good patency at the graft. If a resistive signal is heard with cessation of flow in diastole, this usually signals a technical problem with the anastomosis or it can indicate severe spasm of the donor artery. Under these conditions the anastomosis can be inspected and papaverine can be reapplied to the donor and recipient arteries.
A

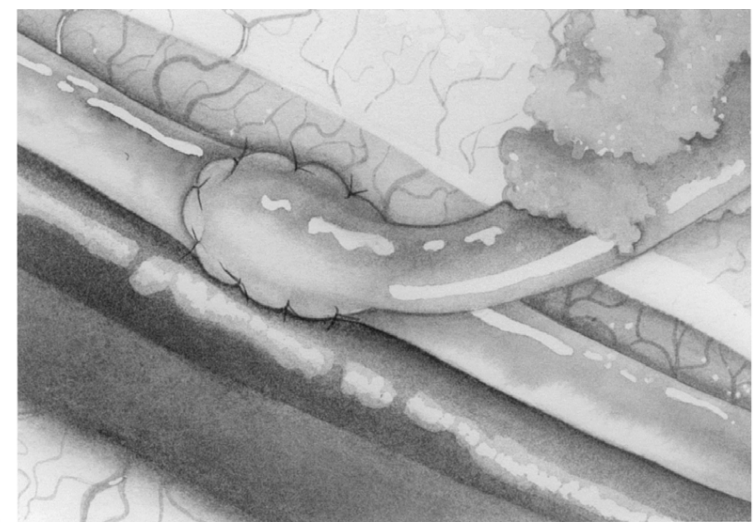

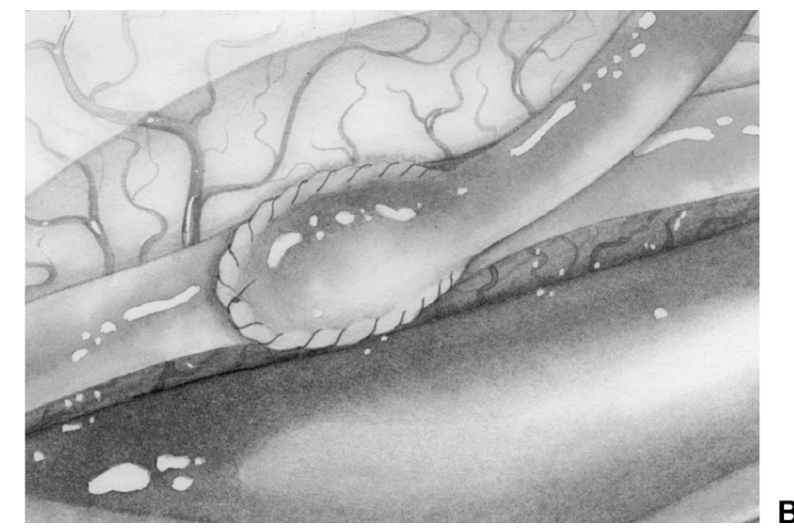

Figure 9 This figure illustrates the two different options of suture closure of the anastomosis using either $(\mathrm{A})$ interrupted or (B) running suture closure. 


\section{DOUBLE-LIMBED STA-MCA BYPASS TO DISTAL SYLVIAN BRANCHES}

In cases where higher flow is anticipated, doublelimbed bypass can be performed to the distal sylvian branches of the MCA. The STA is isolated as previously described, but the cortical dissection is altered slightly. The bone flap that is made is extended more proximally to locate the sylvian fissure at the curvature of the sphenoid wing. Microdissection of the sylvian fissure is accomplished to expose the anterior portion of the insula and locate M2 and M3 branches in the sylvian fissure. These branches are usually free of a large number of perforating vessels, which allows easy mobilization. Two branches of the recipient arteries are isolated and identified, and rubber barriers are placed around the branches. A small amount of retraction is often required at the inferior frontal and superior temporal locations to provide access to the sylvian branches. The microvascular portion of the anastomoses is performed as previously described.

\section{END-TO-END ANASTOMOSIS USING THE MICROANASTOMOTIC DEVICE}

The Microvascular Anastomotic COUPLER System (Synovis Micro Companies Alliance Inc., Birmingham, AL) can be used to perform end-toend anastomoses in cases where double-limbed bypass is utilized. This can provide a slightly higher flow than a standard single end-to-side distal anastomosis and can also be used on more proximal vessels if a large STA is present. ${ }^{13,14}$ The donor artery and the recipient artery are prepared as previously described in the microanastomotic measuring devices used to choose the appropriate ring size. Two of the STA donor branches are mobilized, and instead of being spatulated at the end, they are cut at right angles. The first branch is prepared and loaded in the microanastomotic device. The artery is everted over the six-pin configuration to prepare it for an anastomosis. The recipient artery is then cross-clamped and divided in half, and the other end of the anastomotic wing is placed against the recipient branch. The recipient branch of the MCA is then loaded onto the pins through the ring and inspected. The vessel is thereby perforated from the outer to inner surface. The handle of the microanastomic device is turned allowing anastomosis of the STA branch to the middle cerebral recipient branch. Closing the device brings the two rings into opposition (Fig. 10). Further closure allows each set of pins to become seated in perforations on the opposite ring, and the vessels are coupled with lumen to lumen contact. The two halves of the device are then pinched with forceps to assure
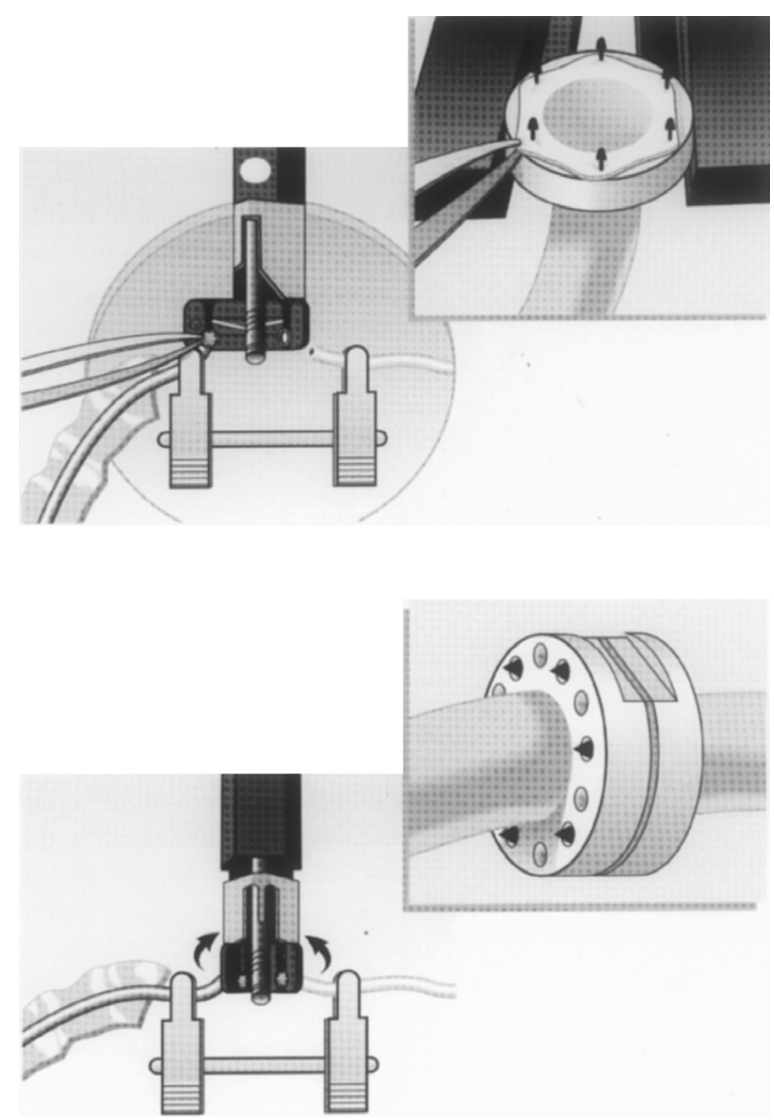

Figure 10 Illustration of the technique of end-to-end vessel anastomosis using the microanastomotic system. Each end of the vessel to be connected is loaded on the polyethelene ring and impaled on the six pins on each ring. The rings are then forced together and the pins of each ring insert into holes in the opposite ring and form a friction fit. 


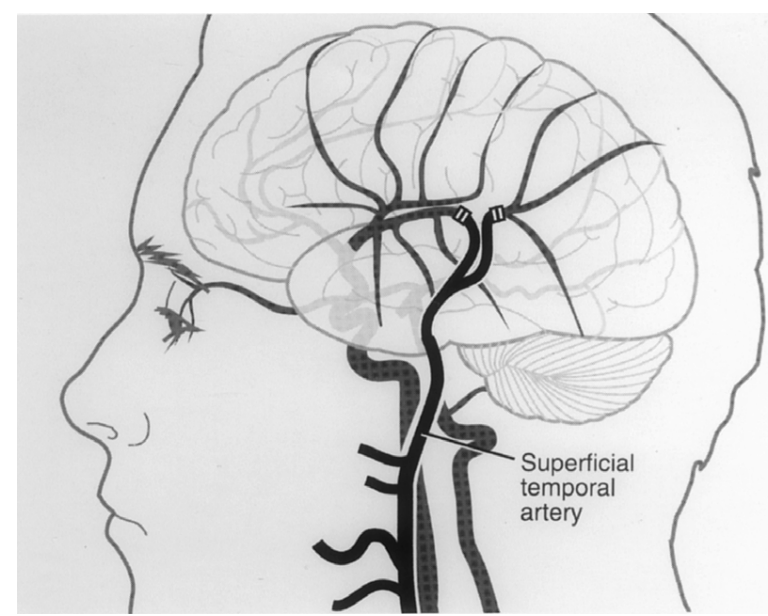

Figure 11 Schematic of the construction of a double limbed STA-MCA bypass from two branches of the STA to a divided branch of the distal MCA. STA, superficial temporal artery; MCA, middle cerebral artery.

proper seating of the pins. Final tightening of the device forces a drive pin to release the vessels and polyethelene rings from the holder. The temporary vessel clips are then released and flow is re-established. This process is then repeated with the second limb of the STA and the other cut end of the MCA branch. The final construction is a two-limbed STA-to-MCA anastomosis providing both distal outflow and proximal inflow (Fig. 11).

\section{SHORT-VEIN BYPASS FROM STA TO MCA}

In patients where the distal STA is not adequate size to perform an anastomosis, it can be isolated proximally at the level of the tragus. The artery is usually $\sim 2.5 \mathrm{~mm}$ in diameter at this point, and a vein interposition graft can be used to perform an anastomosis at this location. The distal anastomosis is performed on an M3 or M2 branch in the sylvian fissure to allow a larger recipient artery for anastomosis. A reversed saphenous vein graph is harvested from the leg in the standard fashion, and the appropriate length is cut from the vein to course from the donor to the recipient artery. The distal

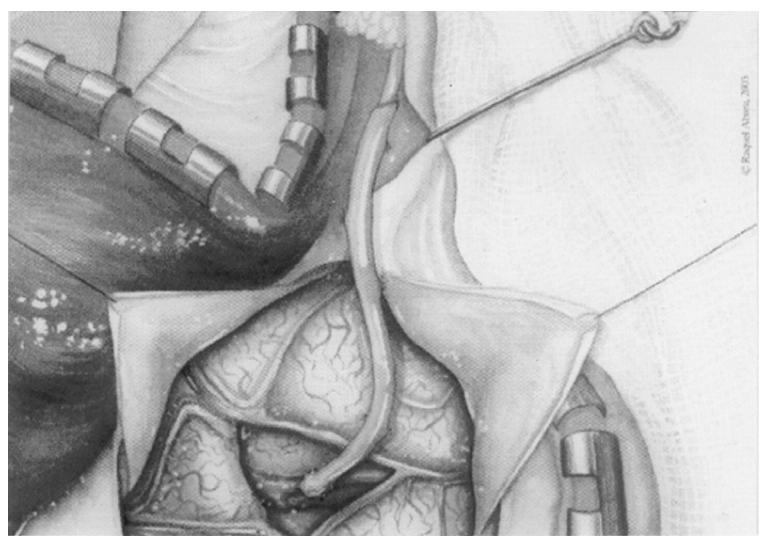

Figure 12 Figure illustrating the course of a saphenous vein graft from the proximal STA (inferior) to a sylvian branch of the MCA (superior). STA, superficial temporal artery; MCA, middle cerebral artery.

anastomosis is performed at the recipient artery in the sylvian fissure using interrupted or running 9-0 sutures with a tapered needle. It is sometimes helpful to perform the recipient artery anastomosis first so the vein can be flipped from side-to-side to sew both sides of the anastomosis. A proximal anastomosis can be performed with an $8-0$ or $7-0$ suture. The donor and recipient arteries are both spatulated and running heel stitches are placed. Running suture technique is used to seal both limbs of the anastomosis (Fig. 12).

\section{CLOSURE OF THE CRANIOTOMY}

It is important when closing the craniotomy to rongeur an adequate portion of bone to prevent compression of the graft by the craniotomy flap. Prior to this step the dura is approximated loosely around the graft to prevent constriction. Following bone flap replacement, the temporalis muscle is reapproximated loosely to prevent any compression. The graft is inspected for kinking or compression during all phases of the closure. Warm saline solution is used to irrigate the graft and dilute papaverine is used to prevent spasm. 


\section{POSTOPERATIVE CARE}

After surgery it is important to prevent compression of the graft site and also to insure adequate blood pressure. The patient's blood pressure is kept above 110 systolic to prevent graft occlusion. On the first postoperative day, patients are begun on aspirin 325 $\mathrm{mg}$ per day to inhibit platelet function.

\section{POTENTIAL PITFALLS AND COMPLICATIONS}

Complications and undesired results have been described following this procedure but most can be avoided with attention to technical details. Some of these unwanted outcomes include graft occlusion, subgaleal hematoma, scalp necrosis, and postoperative intracranial hemorrhage.

It is important to have a technically perfect anastomosis with no damage during dissection of the donor or recipient artery to achieve short- and long-term patency of the graft. It is also equally important to avoid kinking or pressure by the bone edges from inadequate bone removal during closure to prevent graft compromise. If resistive flow is occurring during closure, certain maneuvers can be useful but they will not overcome the effect of a poor technical anastomosis. Maneuvers to salvage flow include maintenance of adequate blood pressure (above $110 \mathrm{~mm} \mathrm{Hg}$ systolic), irrigation with warm, not cold, saline with dilute papaverine, and administration of $500 \mathrm{cc}$ of dextran 40 to decrease platelet function.

Prevention of scalp problems can be achieved by limited lateral dissection of the STA, adequate hemostasis, and postoperative subgaleal drain. Limited cortical dissection and pre- servation of veins is helpful to prevent intracranial hemorrhage.

\section{REFERENCES}

1. Yasargil MG. A legacy of microneurosurgery: memoirs, lessons, and axioms. Neurosurgery 1999;45:1025-1092

2. de Weerd AW, Veldhuizen RJ, Veering MM, Poortvliet DC, Jonkman EJ. Long-term clinical and neurophysiological effects of reconstructive vascular surgery for cerebral ischemia. Acta Neurol Scand 1989;79:311-315

3. Diaz FG, Umansky F, Mehta B, et al. Cerebral revascularization to a main $\operatorname{limb}$ of the middle cerebral artery in the Sylvian fissure. An alternative approach to conventional anastomosis. J Neurosurg 1985;63:21-29

4. Gratzl O, Schmiedek P. STA-MCA bypass: results 10 years postoperatively. Neurol Res 1983;5:11-18

5. Spetzler RF. Retinal circulation after STA-MCA bypass. Neurosurgery 1985;16:583

6. Sundt TM Jr, Siekert RG, Piepgras DG, Sharbrough FW, Houser OW. Bypass surgery for vascular disease of the carotid system. Mayo Clin Proc 1976;51:677-692

7. [No authors listed.]Failure of extracranial-intracranial arterial bypass to reduce the risk of ischemic stroke. Results of an international randomized trial. The EC/IC Bypass Study Group. N Engl J Med 1985;313:1191-1200

8. Srinivasan J, Britz GW, Newell DW. Cerebral revascularization for moyamoya disease in adults. Neurosurg Clin N Am 2001;12:585-594; ix

9. Onesti ST, Solomon RA, Quest DO. Cerebral revascularization: a review. Neurosurgery 1989;25:618-628; discussion 28-29

10. Newell DW, Skirboll SL. Revascularization and bypass procedures for cerebral aneurysms. Neurosurg Clin N Am 1998;9:697-711

11. McCormick PW, Tomecek FJ, McKinney J, Ausman JI. Disabling cerebral transient ischemic attacks. J Neurosurg 1991;75:891-901

12. Grubb RL Jr. Extracranial-intracranial arterial bypass for treatment of occlusion of the internal carotid artery. Curr Neurol Neurosci Rep 2004;4:23-30

13. Newell DW, Dailey AT, Skirboll SL. Intracranial vascular anastomosis using the microanastomotic system. Technical note. J Neurosurg 1998;89:676-681

14. Newell DW, Schuster JM, Avellino AM. Intracranial-tointracranial vascular anastomosis created using a microanastomotic device for the treatment of distal middle cerebral artery aneurysms. Technical note. J Neurosurg 2002;97:486-491 
